\title{
Survey of Candidate Pulsating Eclipsing Binaries - I
}

\author{
S. Dvorak ${ }^{1}$ \\ ${ }^{1}$ Rolling Hills Observatory, Clermont, FL USA
}

\begin{abstract}
Initial results from a photometric survey of stars selected from the list of eclipsing binaries that may contain a pulsating component by Soydugan et al. (2006) are reported. A minimum of two nights of CCD observations with $\mathrm{V}$ and/or $\mathrm{B}$ filters of each of the 35 stars from this list was collected. Of the 35 stars studied, a pulsating component was detected in three of the systems. Pulsations were also serendiptiously detected in the eclipsing binary RR Leporis, which is not on the candidate list.

Accepted: 2009, August 21

Individual Objects: SZ Ari, CG Aur, V0417 Aur, UW Boo, V364 Cas, V Crt, MY Cyg, V456 Cyg, V466 Cyg, V477 Cyg, SZ Her, TXHer, UX Her, KW Hya, UU Leo, VZ Leo, WY Leo, RR Lep, SX Lyn, BO Mon, EP Mon, V501 Oph, EY Ori, FT Ori, V536 Ori, TY Peg, BG Peg, BO Peg, IQ Per, IU Per, ZZ Pup, AC Tau, AQ Tau, EW Tau, RS Tri, KP Vir.
\end{abstract}

\section{Introduction}

Soydugan et al. (2006) introduced a catalog of eclipsing binaries with photometric color or spectroscopic classification that placed one or both components within the $\delta$ Scuti region of the instability strip. These stars were selected from existing catalogs of photometric and spectroscopic data, including a number of little-studied stars discovered by the Hipparchos mission.

Eclipsing binaries, particularly those with total eclipses, are especially useful for testing stellar models. Photometry in different bandpasses combined with radial velocity measurements often make it possible to determine accurate physical parameters for the components. These values can then be compared to star models as a check on the model's accuracy. This check is particularly interesting for pulsating variables where the models have to consider the 
star's internal structure in more detail. In an attempt to find suitable targets for further study a survey of eclipsing binaries possibly containing $\delta$ Scuti-type variables was undertaken at Rolling Hills Observatory ( $\mathrm{RHO}$ ), using the catalog of Soydugan et al. as a source of targets.

\section{Procedures}

\subsection{Selection of Targets}

A selection of stars suitable for observations with the $0.25 \mathrm{~m}$ telescope and $\mathrm{CCD}$ at RHO was taken from this catalog. Stars brighter than $\mathrm{V} \approx 12$ with declinations between -20 and +57 degrees were selected from this list and examined to ensure that suitable comparison stars were available. From the stars that met these criteria 35 were chosen for the first series of observations reported in this paper.

One additional target, RR Leporis, was added to the list selected from Soydugan et al. This star's short-period oscillations were detected during an earlier, unrelated time series taken while observing a primary eclipse of that star.

\subsection{Observation and reductions}

$\delta$ Scuti stars typically have periods ranging from approximately 0.2 to 4 hours so each star was observed for a minimum of 4 hours per night, on at least two nights. The amplitude of pulsations are larger in shorter wavelengths so a $B$ filter was used where target and comparison star brightness permitted (roughly $<8.5 \mathrm{mag})$. Fainter stars were measured with a $\mathrm{V}$ filter. Time series were generally collected between primary and secondary eclipses, near phases 0.25 and 0.75 , to minimize the light variations due to the eclipsing nature of the systems.

All data collection was done with the Meade $0.25 \mathrm{~m}$ Schmidt-Cassegrain telescope and SBIG ST-9XE CCD at RHO, using either a Johnson B or V filter. Image cadence was typically between 35 and 60 seconds, depending on the target's brightness, as a compromise between photometric accuracy and the need for frequent sampling for the short-period oscillations. Where possible, multiple comparison stars were used to reduce the average error. The precision of individual measurements was generally better than 6 millimags.

The images were processed using normal dark and flat frame processing, and star lists were extracted using aperture photometry with sextractor (Bertin \& Arnout 1996). Differential magnitudes for the target and check stars were produced using proprietary software. The nightly differential magnitude measurements for each star was flattened using a polynomial fit to eliminate variations 
due to the eclipsing nature of the systems. Each night's data was shifted to bring the average of each data set into agreement. Data taken during primary or secondary eclipse where the target star's brightness differed from its out of eclipse brightness by more than approximately 0.3 mag were omitted.

\section{Analysis and results}

A period search was conducted with the Fourier analysis routine in Period04 (Lenz \& Breger 2005) on the normalized data for each star. Of the 35 stars selected from the Soydugan list, three showed statistically significant signals with $\mathrm{S} / \mathrm{N}>5$ in the range $6 \geq f \leq 50 \mathrm{~cd}^{-1}$ that is expected for $\delta$ Scuti stars. The results for all 36 stars are shown in Table 1 .

Table 1: Observed Stars

\begin{tabular}{lccl} 
Star & Nights & Freq $\left(\mathrm{cd}^{-1}\right)$ & Comments \\
\hline SZ Ari & 3 & - & - \\
CG Aur & 3 & - & - \\
V0417 Aur & 5 & - & - \\
UW Boo & 4 & - & - \\
V364 Cas & 8 & - & Equal components \\
V Crt & 4 & - & - \\
MY Cyg & 3 & - & - \\
V456 Cyg & 2 & - & - \\
V466 Cyg & 2 & - & - \\
V477 Cyg & 3 & - & - \\
SZ Her & 4 & - & - \\
TX Her & 8 & - & - \\
UX Her & 2 & - & - \\
KW Hya & 2 & - & - \\
UU Leo & 5 & - & - \\
VZ Leo & 5 & - & - \\
WY Leo & 27 & $15.2528(1)$ & - \\
RR Lep & 13 & $31.8654(3)$ & - \\
SX Lyn & 4 & - & - \\
BO Mon & 4 & - & - \\
EP Mon & 5 & - & - \\
V501 Oph & 2 & - & - \\
EY Ori & 7 & - & - \\
& & & Table continued on next page
\end{tabular}




\begin{tabular}{lccl} 
& \multicolumn{3}{c}{ continued from previous page } \\
Star & Nights & Freq $\left(\mathrm{cd}^{-1}\right)$ & Comments \\
\hline FT Ori & 6 & - & - \\
V536 Ori & 4 & - & - \\
TY Peg & 4 & - & - \\
BG Peg & 16 & $24.9857(1)$ & - \\
BO Peg & 3 & - & - \\
IQ Per & 6 & - & Min II is total \\
IU Per & 2 & - & - \\
ZZ Pup & 5 & - & - \\
AC Tau & 9 & $17.533(1)$ & - \\
AQ Tau & 3 & - & - \\
EW Tau & 6 & - & - \\
RS Tri & 4 & - & - \\
KP Vir & 15 & - & Unsolved Hipparchos EB
\end{tabular}

Table 2 presents information on the four systems containing pulsating components found in this study. The table includes observational information including the target star position and comparison and check stars used, as well as physical parameters such as spectral class and $\mathrm{V}$ magnitude obtained from the SIMBAD database.

Table 2: Pulsating Star Details

\begin{tabular}{l|l|c|c|l} 
Star & Position (J2000) & V mag & Sp Type & Comparison Stars \\
\hline WY Leo & $09: 31: 01.1+16: 39: 25.2$ & 11.0 & A2 & GSC 01403-00795 \\
& & & & GSC 01403-01412 \\
RR Lep & $05: 12: 10.5-13: 11: 58.6$ & 9.98 & A4 & GSC 01403-00075 \\
BG Peg & $22: 52: 47.2+15: 39: 09$ & 10.7 & A2 & GSC 01698-000071 \\
AC Tau & $04: 37: 06.4+01: 41: 31.2$ & 10.5 & A8 & GSC 00082-00234 \\
& & & & GSC 00083-00652 \\
& & & & GSC 00083-00713 \\
& & & & GSC 00083-00681
\end{tabular}


Table 3: Orbital and Pulsation Periods

\begin{tabular}{l|l|c|c} 
Star & $\begin{array}{c}\text { Amplitude } \\
\text { (V mag) }\end{array}$ & $\begin{array}{c}\mathrm{P}_{\text {orb }} \\
\text { (days) }\end{array}$ & $\begin{array}{c}\mathrm{P}_{\text {puls }} \\
\text { (days) }\end{array}$ \\
\hline WY Leo & $0.011(1)$ & 4.98578 & $0.0655617(9)$ \\
RR Lep & $0.005(1)$ & 0.91543 & $0.0313820(6)$ \\
BG Peg & $0.015(2)$ & 1.95243 & $0.0400229(3)$ \\
AC Tau & $0.006(1)$ & 2.04340 & $0.057035(7)$
\end{tabular}

Detailed information on the four systems where pulsations were detected are shown in Table 3. The amplitude from Period04, eclipsing binary orbital period, pulsational period from this study, and the ratio of pulsation to orbital period are shown in the Amplitude, $\mathrm{P}_{\text {orb }}, \mathrm{P}_{\text {puls }}$, and $\mathrm{P}_{\text {puls }} / \mathrm{P}_{\text {orb }}$ columns, respectively. Errors from the least-squares analysis in Period04 are included in parentheses for the amplitude and $P_{\text {puls }}$ values. Figures 1 through 4 show the power spectrum for each of the four systems. Representative single-night unflattened light curves for these systems are shown in Figures 5 through 8.

The three positive detections out of 35 targets equals a success rate of $8.6 \%$. All of the systems where pulsations were detected consisted of components that are quite unequal in brightness which made detecting pulsations, presumably in the brighter component, far easier. In systems where the components are close in brightness, such as V364 Cas, or where the fainter star is the pulsator, the detection of pulsations would necessarily be more difficult. Of the 32 systems where no pulsations were detected it is certainly possible that a pulsating component does in fact exist but the pulsations were too small to be detected by the instruments used in this study. In addition, $\delta$ Scuti stars often exhibit multiple, interfering pulsation frequencies, and additional monitoring of these systems might detect pulsations when these frequencies add constructively.

Liakos, A. \& Niarchos, P. (2009) contains a survey of 24 stars similar to the current paper. Three of that paper's stars were also studied in the current paper: UW Boo, V456 Cyg, and V466 Cyg. The results in that paper are in agreement with the current results in that no pulsations were detected in any of these three stars. Liakos \& Niarchos detected pulsations in three stars, or $12.5 \%$. 


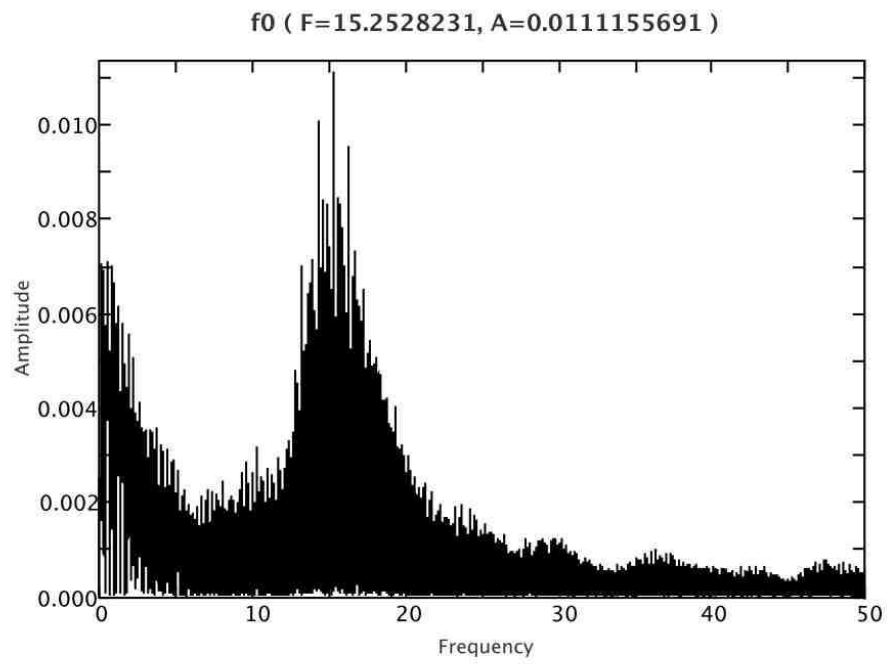

Figure 1: Periodogram of WY Leo

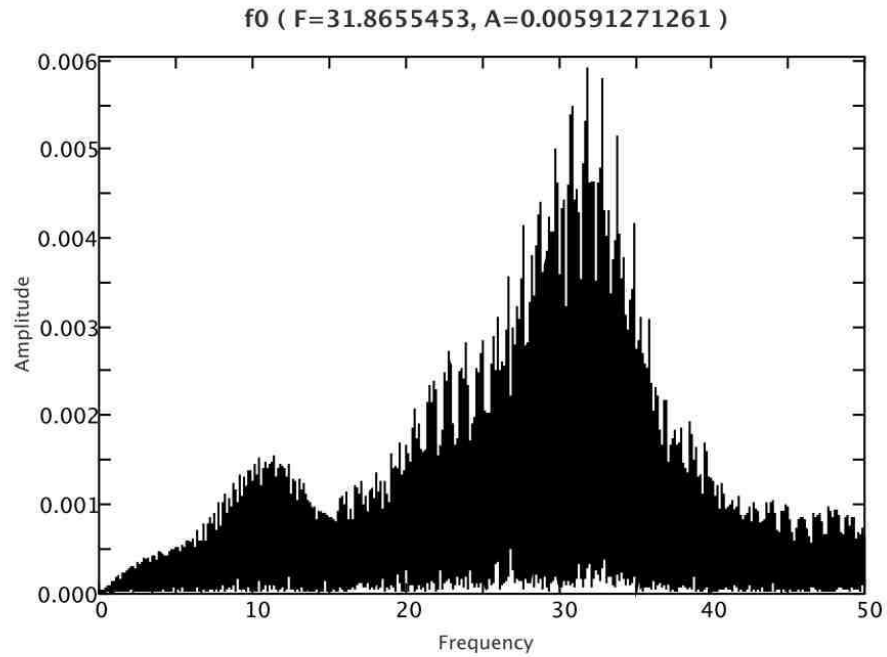

Figure 2: Periodogram of RR Lep 


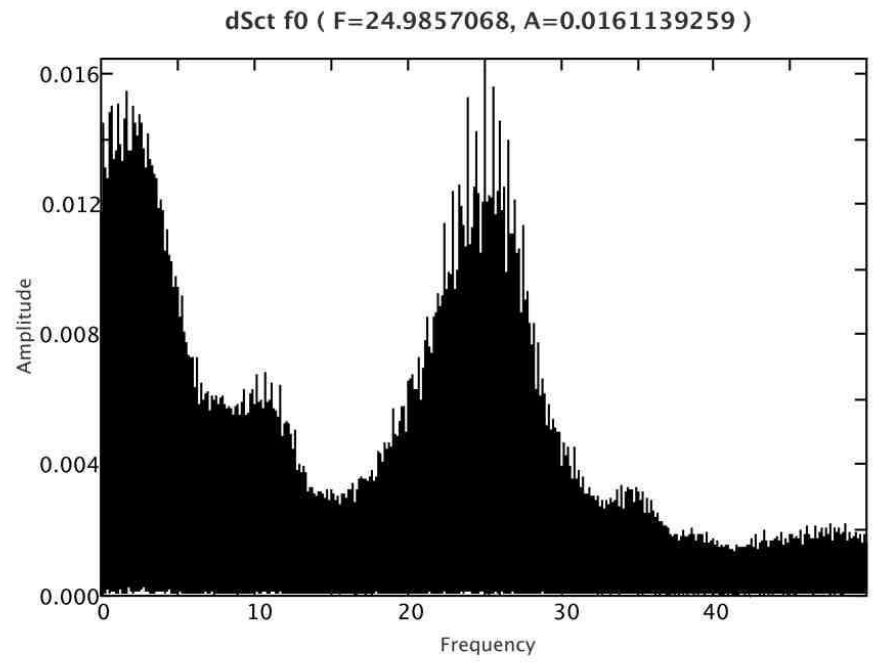

Figure 3: Periodogram of BG Peg

fo ( $F=17.53447, A=0.00527258306$ )

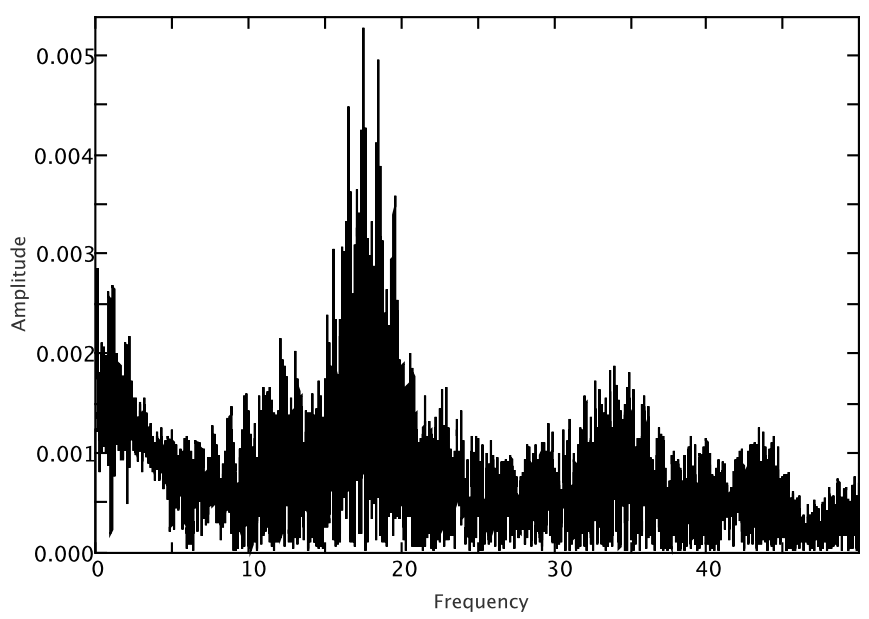

Figure 4: Periodogram of AC Tau 


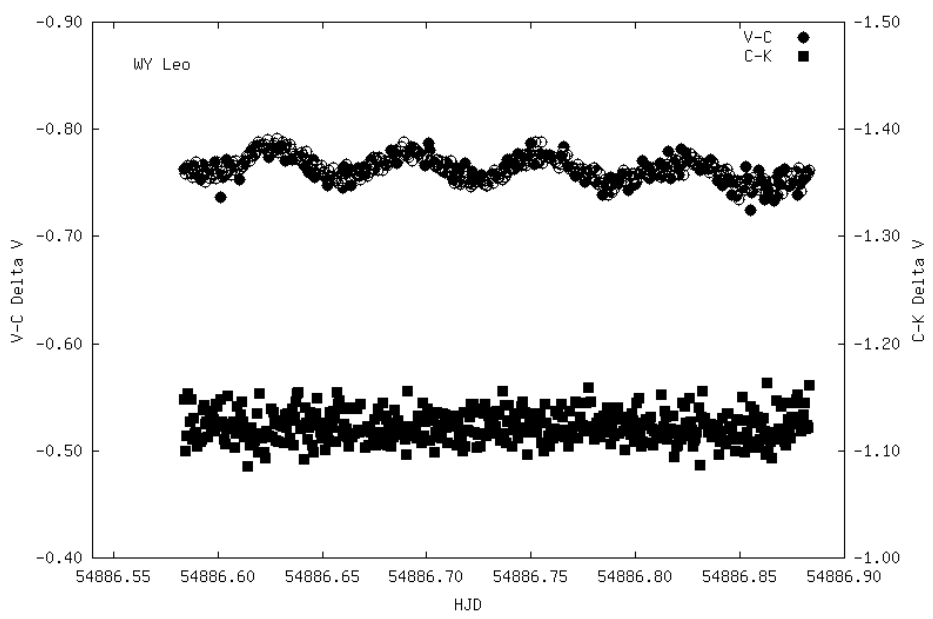

Figure 5: Single-night differential V light curve of WY Leo

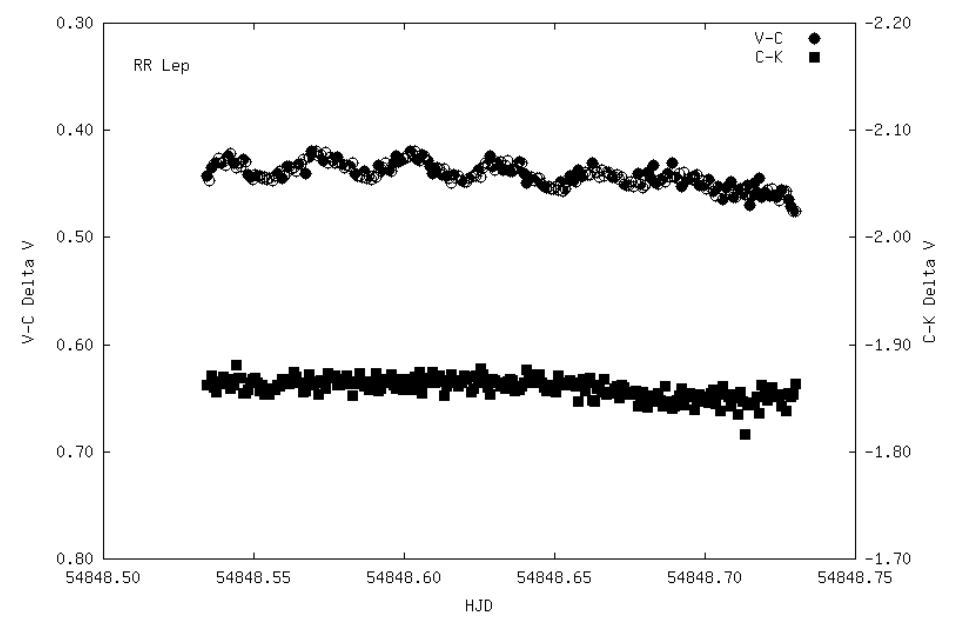

Figure 6: Single-night differential V light curve of RR Lep 


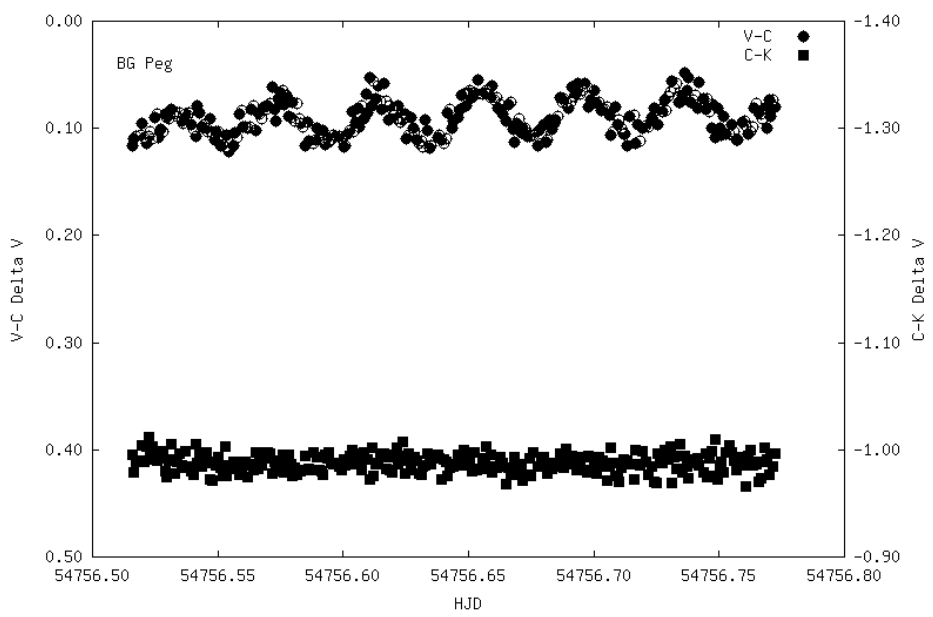

Figure 7: Single-night differential V light curve of BG Peg

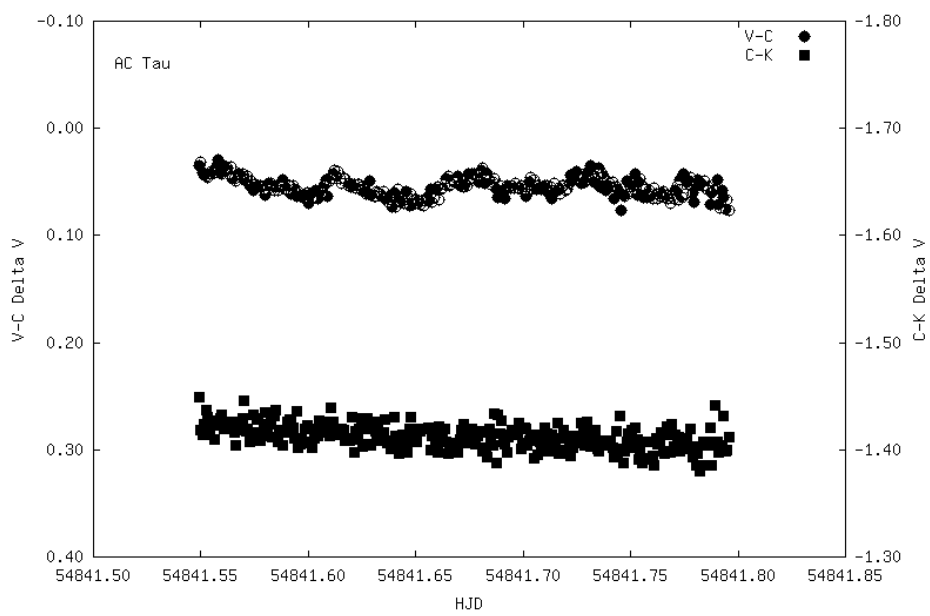

Figure 8: Single-night differential V light curve of AC Tau 


\section{Conclusions}

$\delta$ Scuti stars in eclipsing binary systems can be a useful tool for constraining stellar models. Of 35 eclipsing binaries in this study that potentially contain such stars selected from Soydugan et al. (2006) three (8.6\%) were found to have a pulsating component. The remaining 32 showed no statistically significant variations in the Fourier analysis within the frequency range expected for $\delta$ Scuti stars. This detection rate is consistent with the $12.5 \%$ rate reported by Liakos \& Niarchos (2009). One additional eclipsing system containing a pulsating component, RR Leporis, was previously discovered at RHO and was included in the paper.

Acknowledgments. This research has made use of the SIMBAD database operated at CDS, Strasbourg, France

\section{References}

Bertin, E., \& Arnout, S. 1996, A\&AS, 117, 393

Lenz, P. \& Breger, M. 2005, CoAst, 146, 53

Liakos, A. \& Niarchos, P. 2009, CoAst, 160, 2

Soydugan, E., Soydugan, F., Demircan, O., \& Ibanoglu, C. 2006, MNRAS, 370, 2013 